http://www.jfas.info

\title{
CLASSIFICATION OF VISUALIZATION EXUDATES FUNDUS IMAGES RESULTS USING SUPPORT VECTOR MACHINE
}

\author{
H. A. $\operatorname{Hassan}^{1,2}$, N. M. Tahir ${ }^{1, *}$, A. Zabidi ${ }^{1}$, I. M. Yassin ${ }^{1}$ and M. Karbasi ${ }^{3}$ \\ ${ }^{1}$ Faculty of Electrical Engineering, Universiti Teknologi MARA, 40450 Shah Alam, Selangor, \\ Malaysia \\ ${ }^{2}$ Faculty of Engineering, Universiti Selangor, 45600 Bestari Jaya, Selangor, Malaysia \\ ${ }^{3}$ Kulliyyah of Information and Communication Technology, International Islamic University \\ of Malaysia, 50728 Kuala Lumpur, Malaysia
}

Published online: 05 October 2017

\begin{abstract}
This paper classifies the characteristics of normal and exudates fundus images by determine its accuracy for diagnostic purposes. Image normalization was performed on 149 fundus images (81 normal and 68 exudates) from MESSIDOR databases to standardize the colours in the fundus images. The OD removed fundus image and fundus image with the exudates areas removed. The SVM1 classifier was applied to 30 test fundus images to determine the best optimal parameter. The kernel function settings; linear, polynomial, quadratic and RBF have an effect on the classification results. For SVM1, the best parameter in classifying pixels is linear kernel function. The visualization results using CAC and radar chart are classified using SVM2 to determine its accuracy. It has proven to discriminated exudates and non exudates pixels in fundus image using linear kernel function of SVM1 to diagnose DR.
\end{abstract}

Keywords: Diabetic retinopathy (DR); Optic disc (OD); Support Vector Machine (SVM); Colour Auto Correlogram (CAC); Radial Basis Function (RBF).

\footnotetext{
Author Correspondence, e-mail: nooritawati@ieee.org doi: http://dx.doi.org/10.4314/jfas.v9i4s.2
} 


\section{INTRODUCTION}

Diabetes mellitus affects ten of million people around the world. The statistics indicate that the number will double in the future [1]. The disease is divided into two categories; namely Type I (insulin-dependent) and Type II (insulin-independent) [2-3]. Type I means the immune system destroyed beta cells that make insulin and cause the body to make little insulin or no insulin $[4,5]$. It usually happens in children and teenagers. Meanwhile, Type II stands for person who is insulin resistance, body may not make enough insulin or both [5]. It usually occurs in people who are older or overweight $[4,6]$. Type II is results from interaction between lifestyle, genetic and medical conditions [6]. In reality, almost $80 \%$ of people with Type II are overweight. However, nowadays children and teenagers are exposed to Type II according to obesity and inactiveness $[4,6]$.

Symptoms of diabetes mellitus include delayed wound healing, infection of the urinary system, numbness, thirst, frequent urination and fatigue, as well as weight loss [3]. Due to the generic nature of the symptoms, the disease has the tendency to be detected at advanced stages [3]. Thus, late detection of diabetes is common in Malaysia [7], as well as other parts of the world. Long standing diabetes mellitus has high potential to get diabetic retinopathy (DR) [8-10]. For Type I disease, 90\% have DR after 10 to 15 years [8]. Also, about 25\% of Type I patients develop proliferative diabetic retinopathy (PDR) after 15 years of diabetes mellitus [9]. Meanwhile for Type II disease, the rates of patients who have DR increase to more than $80 \%$ by 15 to 19 years. As well as PDR usually occurs after 25 years of diabetes mellitus [8].

Generally, retina fundus images of diabetic patients exhibit exudates, which are lesions indicative of Diabetic Retinopathy (DR). Therefore, the fundus images can be used to assist ophthalmologists and medical doctor for the early screening of the disease with the assistance of modern computational methods.

This research proposes a method for graphical representation of fundus images to simplify detection of diabetes. The method consists of capturing several key features of the Color Auto Correlogram (CAC) extracted from fundus images, and plotting them in the form of radar chart. The method is centered on the detection and removal of exudates from the original fundus 
image, then comparing the $\mathrm{CAC}$ features of the original image with the exudate-removed image. If exudates are present, the CAC features exhibit significantly different features, which can be easily, distinguished using the radar chart. The CAC and radar chart features were then classified using another SVM classifier.

\section{LITERATURE REVIEW}

\subsection{Diabetes and Diabetic Retinopathy (DR)}

According to International Diabetes Federation Malaysia, there were 3.3 million cases of diabetes in Malaysia in 2015 [11]. DR is found to be the frequent cause of blindness. Early diagnosis can prevent visual loss and blindness but most of diabetic patients had no awareness to perform eye checkup [12].

All individual with diabetes (Type I and Type II) and pregnant women with diabetes are at risk for this disease [13-14]. Symptoms of DR included blurring of vision, difficulty reading, a sudden loss of vision in one eye, seeing rings around lights and dark spots or flashing lights [15-16].

The disease are divided into two categories namely Non-Proliferative Diabetic Retinopathy (NPDR) and Proliferative Diabetic Retinopathy (PDR) [14]. NPDR is the early stage of DR and if the patient blood sugar is uncontrolled, it will rise to PDR. NPDR is characterized by the appearance of deposits of cholesterol (exudates) in the retina can be later be developed into bleeding (hemorraghe) $[13-15,17]$. However, early diagnosis and treatment from NPDR can prevent PDR from damaging the patient's vision [13].

There are many tissues present in the eye but the most important tissue is the retina. The retina is a light-sensitive layer at the back of the eye. The retina changes the light into nerve signals and transmits it to the brain via the optic nerve [18-19]. DR is one of the diseases in the retina which caused several abnormalities such as microaneurysms, exudates, macular edema and hemorraghes.

\subsection{Fundus Image and MESSIDOR Database}

A fundus image is an of the eye's interior surface, which includes the retina, optic disc, macula, fovea and posterior pole [20-21]. The fundus image of the retina is basically acquired 
with the digital fundus camera [12] as shown in Fig. 1. The fundus image from MESSIDOR database are acquired using a color video 3CCD camera on a Topcon TRC NW6 non-mydriatic retinograph with a 45 degree field of view [22-26].

The Optic Disc (OD) is the brightest region of fundus image [27]. Since the OD has characteristics similar to the exudates, they may be falsely classified as lesions [28]. Therefore, removal of the OD would improve the classification of the remaining exudate region [24, 29-30].

\subsection{Works on Detection of Disease Using Fundus Images}

In [31], authors presented automated detection of exudates in retinal images using a split and merge algorithm. About 17 exudates fundus images from MESSIDOR database are used in this paper. The split and merge algorithm is applied after OD elimination. The image is separated into square sub regions until similarity is verified. Then, it is merged to neighbouring sub regions that meet some uniformity criterion. The sensitivity and specificity of exudates detection are $89.7 \%$ and $99.3 \%$. Meanwhile, in [10], the morphological operation and connected component analysis are used to remove OD. Tresholding is used to separate the exudates from fundus image. Then, Fuzzy Inference System (FIS) is used to classifying exudates and non exudates. The results found sensitivity and specificity and accuracy of detecting exudates as $91.11 \%, 100 \%$ and $93.84 \%$ respectively.

According to [25], fundus images were used to diagnose three stages of Diabetic Macular Edema (DME). DME can be evaluated by detecting exudates in fundus images. A total of 88 fundus images from publicly available MESSIDOR database are used for this experiment; 41 normal fundus images and $47 \mathrm{DME}$ fundus images. Features extracted using the marker controlled watershed method was classified automatically using the Early Treatment Diabetic Retionpathy Study (ETDRS) grading scale. They reported overall sensitivity, specificity and accuracy of $80.9 \%, 90.2 \%$ and $85.2 \%$.

In [32] reported the method for detecting hard exudates in fundus images by using Support Vector Machine (SVM). The regions into exudates and non- exudates classes were represent using relevant and significant features to get the best separability for classification; colour, size, edge strength and texture. The OD regions were removed to prevent false-positive 
exudates issue. The results found the accuracy of training and testing data for SVM classifier are $92.77 \%$ and $89.9 \%$.

In [33], region of interests (ROIs) was applied for the analysis of texture micro patterns to detect hemorrhages and microaneurysms (HMAs). Local Binary Pattern (LBP) extracted texture features of ROIs before employed SVM for classification. The proposed method yields $87.48 \%$ sensitivity and $85.99 \%$ specificity.

\subsection{Visualization}

Visualization has been developing since Ivan Sutherland had introduced sketchpad (robot draftsman) in 1963 to show that computer graphics could be used for both artistic and technical purpose [34].

When the United States National Science Foundation (NSF) meet formally conference on recent and future growth of computing visualization, the term visualization as a professional term officially began in February 1987 [35].

The objective of visualization is to explore the data and information to gain knowledge [36]. Furthermore, information visualization need to be balance between form and function rather than beautiful data visualizations that failed to give information [37]. The research stems from the unique ability of the human visual system that can detect interesting features and patterns in a short amount of time in visualization data [38].

The main point of visualization is it should use minimal amount of resources to be effective and efficient [38-39]. Hence, good visualization techniques can highlight similarities and differences, show correlations and trends to provide the perceptivity in this process [39].

Visualization algorithms have been designed by engineers and researchers to map the data into a visual form or structure; bar charts, scatter plots, line graphs and many more [34]. In [40] found that most of visualization sources in scientific publications are diagrams, graphs, charts, plots, flowcharts, timelines and tables.

\subsection{Color Auto-Correlogram (CAC)}

Recently several approaches have done to combine color with spatial information [41-44]. The spatial correlation of pairs of colors changes with distance is called color correlogram [45]. Meanwhile spatial correlations between identical color only is called CAC, which is 
subset of color correlogram [45-46]. There are several issues of using color correlogram such as expensive cost of memory space (use in a huge database), computation time and space complexity [43]. Therefore, CAC is applied to reduce the time and space complexity by finding spatial correlations between identical color only [42-43].

\section{METHODOLOGY}

A total of 149 images from the publicly available MESSIDOR database were used for classification and visual the proposed method. The images labeled as are acquired using a color video 3CCD camera on a Topcon TRC NW6 non-mydriatic retinograph with a 45 degree field of view [22].

There are two flowcharts for this experiment; Experiment I: To determine the optimal parameter SVM1 classifier and Experiment II: To visualize exudates using CAC and radar chart. The results also have been verified using SVM2 classifier. The proposed flowcharts are shown in Fig. 1 and Fig. 2.

Several preprocessing steps were performed to normalize the appearance of the fundus images, as well as remove the optic disc that may interfere with the classification process. This step is described in section 3.1 .

The experiment in section 3.2 was to design and implement SVM1 classifier for feature extraction to extract exudates and non-exudates pixels. This was done by collecting samples of exudates and non-exudates pixels to train the SVM1 classifier subject to different kernel functions. After training was completed, the classifiers were both applied to 30 test fundus images to determine the best kernel function for it. The results also have been verified using SVM2 classifier. 


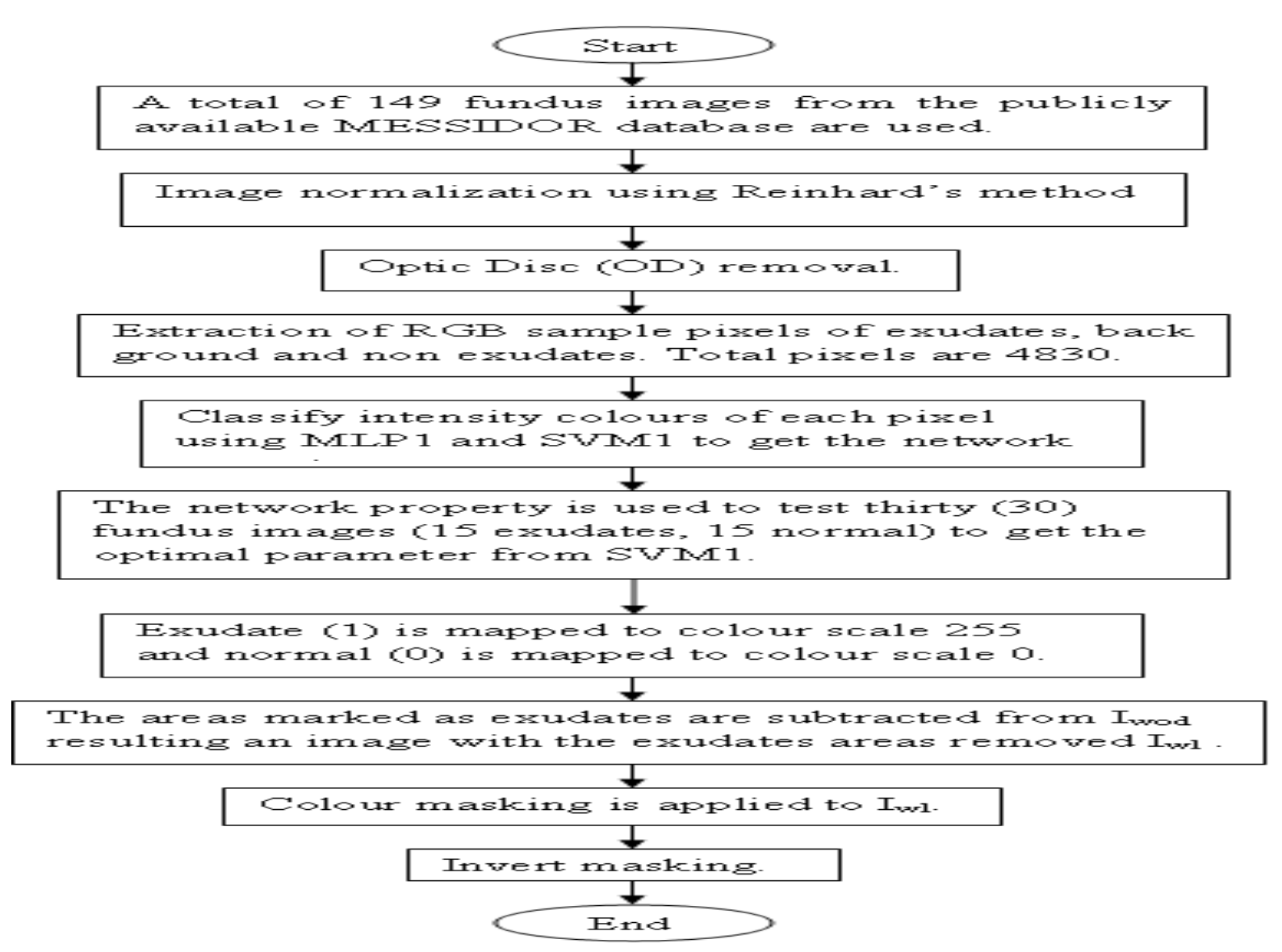

Fig.1. Experiment I: To determine the optimal parameter SVM1

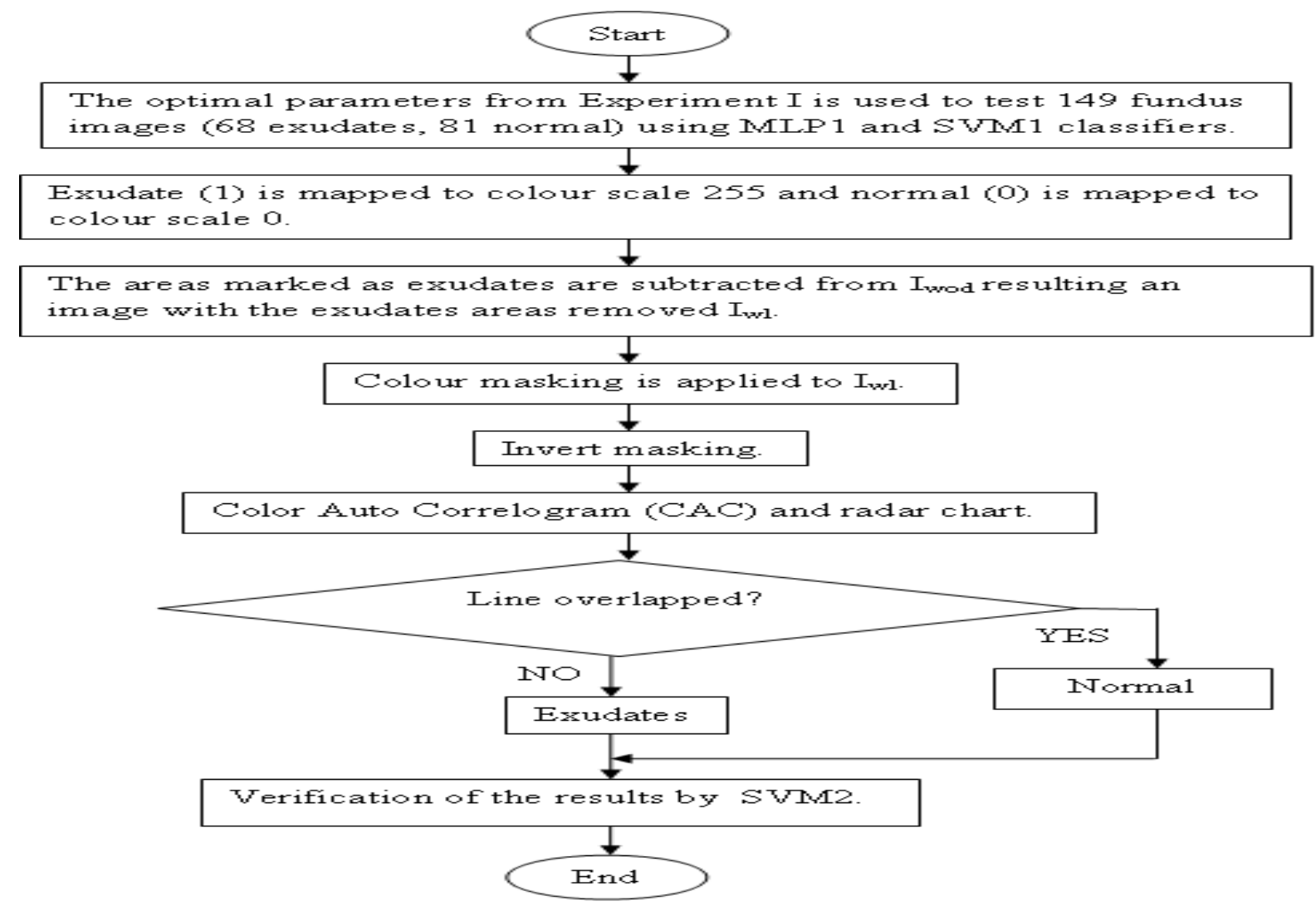

Fig.2. Experiment II: To visualize exudates using CAC and radar chart 


\subsection{Pre Processing: Image Normalization and Removal of OD}

The first step in our approach is to normalize using the histogram equalization method. This is done by first examining the color histogram of the image, then using the histogram to help balance the intensities of the colors in the image based on a predefined reference image $\left(\mathrm{I}_{\text {ref }}\right)$ as shown in Fig. 6 (i). This is to ensure that all the images in the dataset is consistent prior to Optic Disc (OD) removal. The normalized images are referred to as $\mathrm{I}_{\text {norm. }}$. An example of this process is shown in Fig. 6.

Since the OD has characteristics similar to exudates, they may be falsely classified as lesions. Therefore, removal of the OD from $\mathrm{I}_{\text {norm }}$ would improve the classification of the remaining exudate regions. The OD is excluded from all images by marking the center of the OD region in $\mathrm{I}_{\text {norm, }}$ and expanding the circle radius manually until the entire OD has been covered. An example of this process is shown in Fig. 6(iii). For convenience, images with removed OD are referred to as $\mathrm{I}_{\text {wod. }}$

\subsection{Discrimination of Exudates and Non- Exudates Pixels Using SVM1}

This step is concerned with constructing an optimal classifier to discriminate between exudate and non-exudate pixels based on their colour. To achieve this, samples of exudate, non-exudate and background pixels were obtained from several randomly selected fundus images. A total of 4,830 pixels were chosen manually from the selected fundus images as training data for the MLP and SVM. From that number, 2,696 pixels represented exudates while 2,134 pixels represented non-exudates and background. Sample of six test fundus images (3 exudates and 3 normal) from the total 30 test fundus images as shown in Fig. 3 were selected from the full dataset and these images were used to assist in the search for optimal parameters for the classifiers. The SVM1 training process are described in section 3.2.1 respectively. The results of this section are presented in section 4 . 


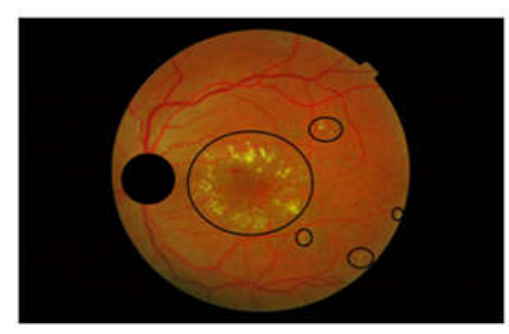

(i) Image 29

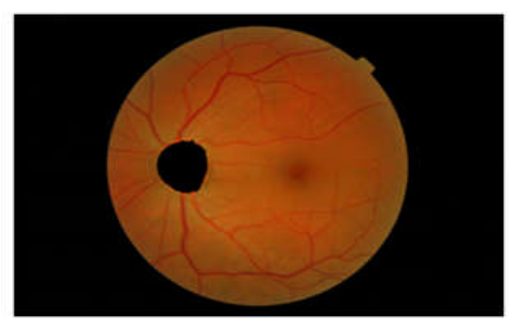

(iv) Image 11

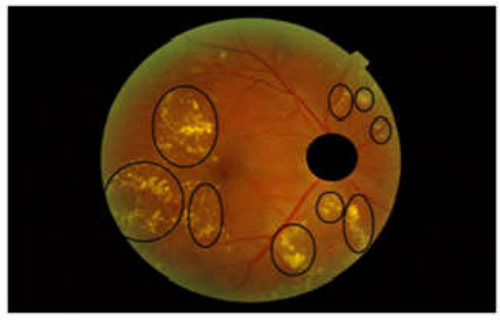

(ii) Image 131

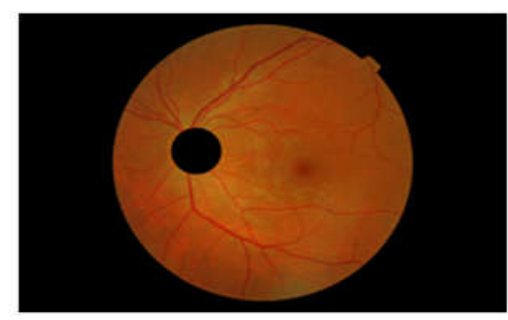

(v) Image 15

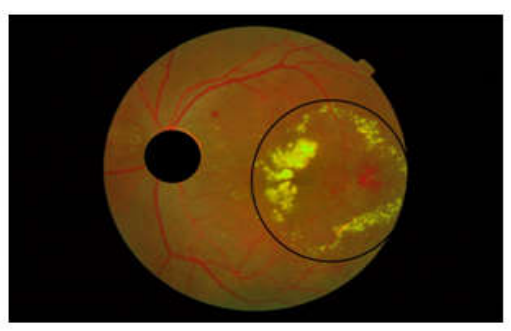

(iii) Image 175

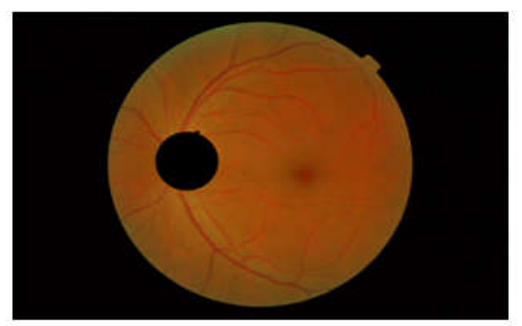

(vi) Image 17

Fig.3. Sample of six fundus images ( 3 exudates, 3 normal) from the total 30 test fundus images

\subsubsection{SVM1 Implementation}

The SVM was trained using samples of exudate and non-exudate pixels. Several kernel structures were tested: linear, polynomial, quadratic and RBF. The inputs for the SVM are Red, Green and Blue (RGB) values of each pixel. The SVM generated a binary output that indicates whether the pixel under examination is exudate or vice versa. The value of $\mathrm{C}$ was varied from $0.01,0.1,1$ and 10 to get the best accuracy test as shown in Table 1 .

The division of training and testing set were 50\% and 50\%; 2415 pixels for training and 2415 pixels for testing. The best model of SVM depends on support vector, C and kernel function parameters. Table 1 shows the SVM1 structure and parameter settings used.

Table 1. SVM1 structure and parameter settings

\begin{tabular}{cc}
\hline Parameter & Setting \\
\hline Kernel functions & Linear, polynomial, quadratic and RBF \\
Regularization parameter & $\mathrm{C}=1$ \\
Division of training and testing set $(\%)$ & $50: 50$ \\
\hline
\end{tabular}

Then, the optimal parameters from SVM1 classifiers are applied to test all $149 \mathrm{I}_{\text {wod }}$ fundus images; 68 exudates and 81 normal fundus images.

\subsection{Visualization Using CAC and Radar Chart}

The CAC method needs to go through all the neighbours for each pixel in the image. It is an 
extension of the color histogram that adds a dimension to the feature vector which represents the relative local distance $\mathrm{k}$ between pixels with the specific colour.

Visualization was performed by extracting CAC features from $\mathrm{I}_{\mathrm{wod}}$ and invert mask $\mathrm{I}_{\mathrm{wl}}$ from exudates fundus image and normal fundus image. The samples of $\mathrm{I}_{\mathrm{wod}}$ and invert mask $\mathrm{I}_{\mathrm{wl}}$ for exudates and normal fundus images are shown in Fig. 4 and Fig. 5.

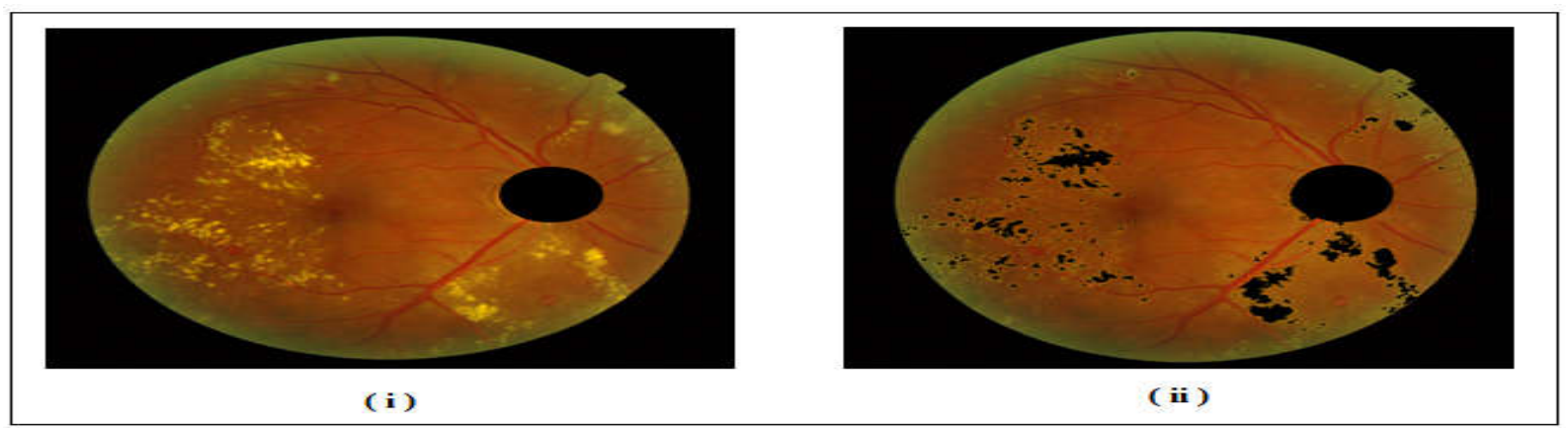

Fig.4. Sample of exudates fundus image for (i) $I_{w o d}$ and (ii) Invert mask $I_{w l}$

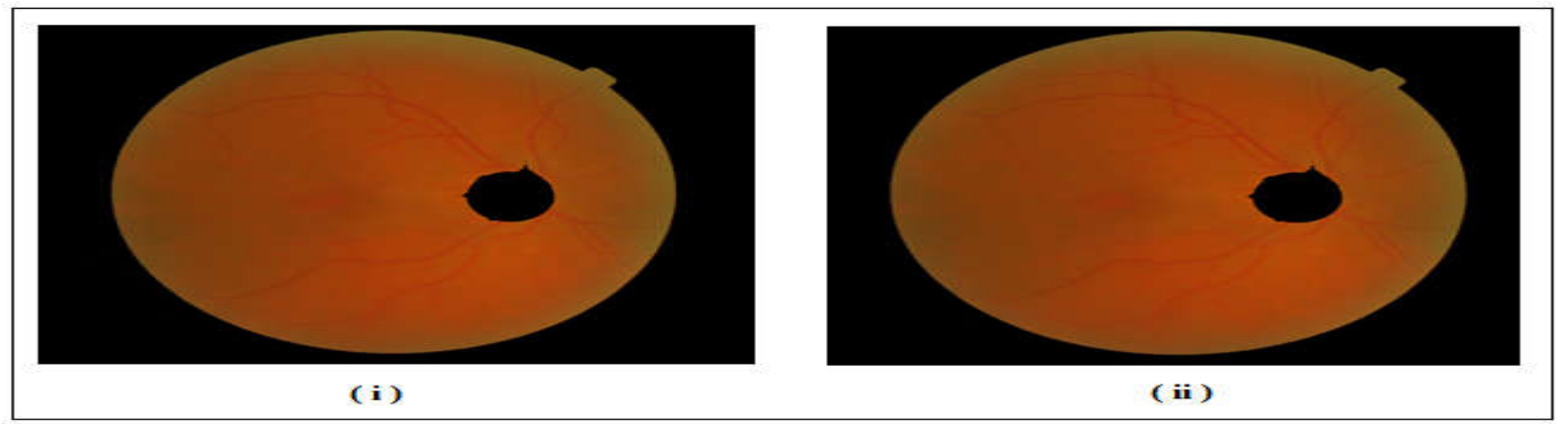

Fig.5. Sample of normal fundus image for (i) $I_{w o d}$ and (ii) Invert mask $I_{w l}$

The inputs for the CAC are image in unsigned 8 bit integers (uint8) form that representing the color image and distance vector, which representing the different distances in which the color distribution is calculated. The output for the CAC is the correlogram vector, which is a straight vector representing the probabilities of occurrence of 64 quantized colors. Its total dimension is $64 \mathrm{n} \times 1$ where $\mathrm{n}$ is the number of different distances. Once the CAC features were obtained from $I_{w o d}$ and invert mask $I_{w l}$, they were plotted on the same radar chart to visualize the features. If exudates are present, they would have been removed from invert mask $I_{w l}$. This in turn changes the shape of the feature plot in the radar chart, which is distinguishable when shown together with the $I_{\text {wod }}$ feature plot. This would indicate a potential diabetic condition. However, if exudates are not present in $I_{w l}$, the feature plot for $I_{w l}$ would show significant overlaps when compared with the $I_{\text {wod }}$ feature plot as both features would essentially be similar or almost similar and the patient is is normal. The results of this section 
are presented in section 4 .

\subsection{Classification Using SVM2}

The input layer was fed with subtraction of values from invert mask $I_{w l}$ minus $I_{w o d}$ fundus images. The SVM2 generated a binary output that indicates whether the CAC features under examination is exudate or vice versa. The best model of SVM depends on support vector, C and kernel function parameters. Several kernel structures were tested: linear, polynomial, quadratic and RBF. The reason of tested several kernel functions is to get the best accuracy for classification. The smallest number of support vector indicates good classifier, which is least number of the difficult data point [47]. The value of $C$ was varied from $0.01,0.1,1$ and 10 to get the best accuracy. The division of training and testing sets were set to 50:50.

\section{RESULTS AND DISCUSSION}

Experimental analysis and testing is run on a Toshiba Satellite laptop Intel Core i7 Central Processing Unit (CPU), 2.1 (GHz) with 4 GB of Random Access Memory (RAM). Window 7 was installed as the operating system. All programs were implemented using MATLAB version 8.1.0.604.

\subsection{Image Normalization and Removal of OD}

The normalization and removal of OD results are presented here. The normalization and removal of OD results are presented here Fig. 6. Fig. 6(i) shows the reference image $\left(I_{\text {ref }}\right)$. Fig. 6(ii) shows before normalization exudates fundus image. Meanwhile, Fig. 6(iii) shows a result of normalization fundus image with OD removal $\left(\mathrm{I}_{\mathrm{wod}}\right)$. Both normalization and OD removal were applied for all 149 fundus images in the dataset.

Fig. 7 shows the color histogram for reference image $\left(\mathrm{I}_{\mathrm{ref}}\right)$, before normalization image and after the Reinhard Stain normalization $I_{\text {norm }}$ and its color histogram have changed as a result of the normalization process. 


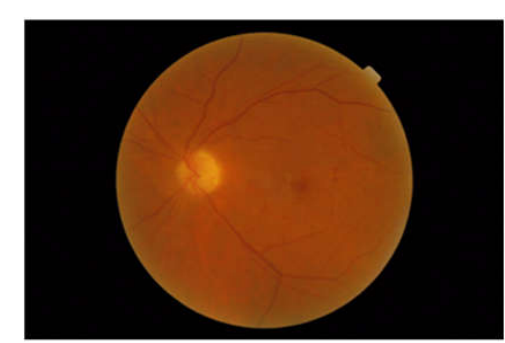

(i)

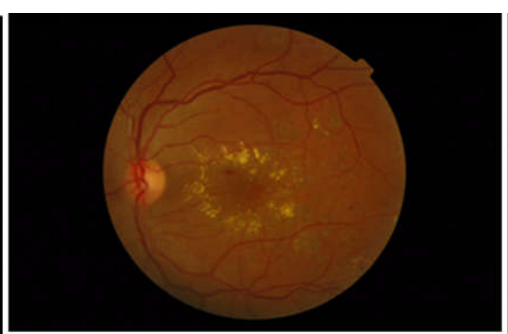

(ii)

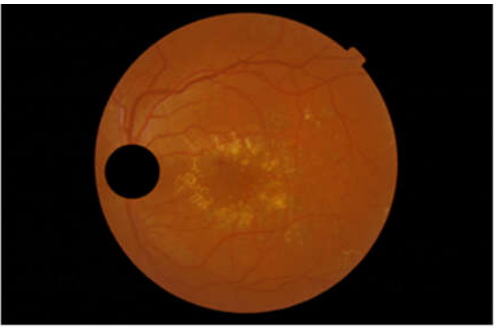

(iii)

Fig.6. (i) The reference fundus image ( $\left.\mathrm{I}_{\mathrm{ref}}\right)$, (ii) Exudate fundus image before normalization, and (iii) Result after normalization and manual OD removal $\left(\mathrm{I}_{\mathrm{wod}}\right)$
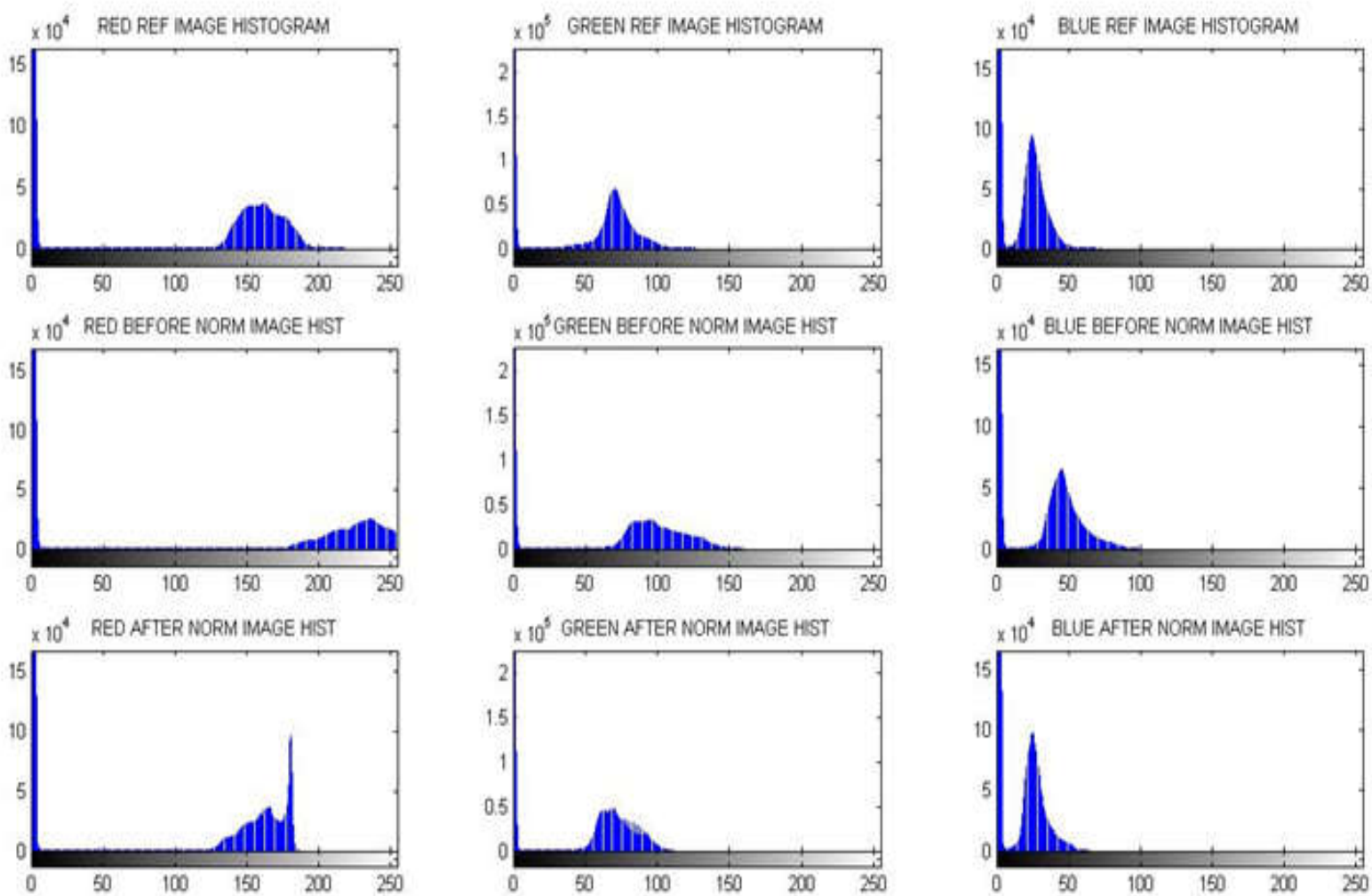

Fig.7. Color Histogram before and after normalization of fundus images

\subsection{Discrimination of Exudates and Non Exudates Pixels Using SVM1 Classifier}

The SVM1 classifier was applied to 30 test fundus images to determine the best optimal parameters for it. A summary of classification results for SVM1 is shown in Table 2 and Table 3. According to Table 2, it shows classification accuracy results of varied $\mathrm{C}$ values. The value is varied from $0.01,0.1,1$ and 10 to get the best classification accuracy to be used in SVM1. From Table 2, the suitable value for $\mathrm{C}$ is equal to 1 since it accuracy was $98.9 \%$ which is the highest value among the values tested.

Four types of kernel functions were applied for pixels classification test; linear, polynomial, 
quadratic and Radial Basis Function (RBF). As can be seen, all the kernel functions show excellent classification accuracy which is above $97 \%$ but to find the best optimal parameter among them, they were applied on six fundus images for exudates segmentation to investigate their performance.

Sample classification results for SVM1 (Fig. 8-Fig. 13) are shown for $\mathrm{I}_{\text {wod }}$ in Fig. 3(i) until Fig. 3(vi).

Table 2. Regularization parameter (C) and classification accuracy for SVM1

\begin{tabular}{cc}
\hline Regularization Parameter $(\mathbf{C})$ & Classification Accuracy \\
\hline 0.01 & $96.7 \%$ \\
0.1 & $97.8 \%$ \\
1 & $98.9 \%$ \\
10 & $95.9 \%$ \\
\hline
\end{tabular}

Table 3. SVM1 kernel functions and pixels classifications

\begin{tabular}{cc}
\hline Kernel Function & Classification Accuracy \\
\hline Linear & Training $=97.0 \%$ \\
& Testing $=95.9 \%$ \\
Polynomial & Training $=100 \%$ \\
& Testing $=100 \%$ \\
Quadratic & Training $=99.6 \%$ \\
& Testing $=99.6 \%$ \\
RBF & Training $=99.3 \%$ \\
& Testing $=99.1 \%$ \\
\hline
\end{tabular}




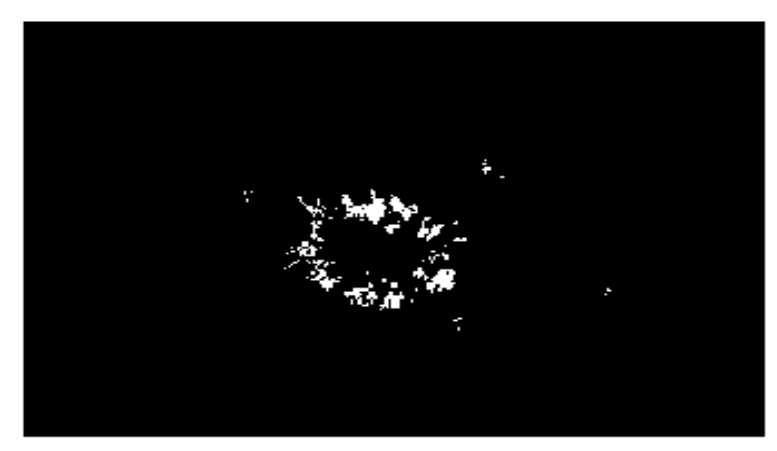

(i) Linear

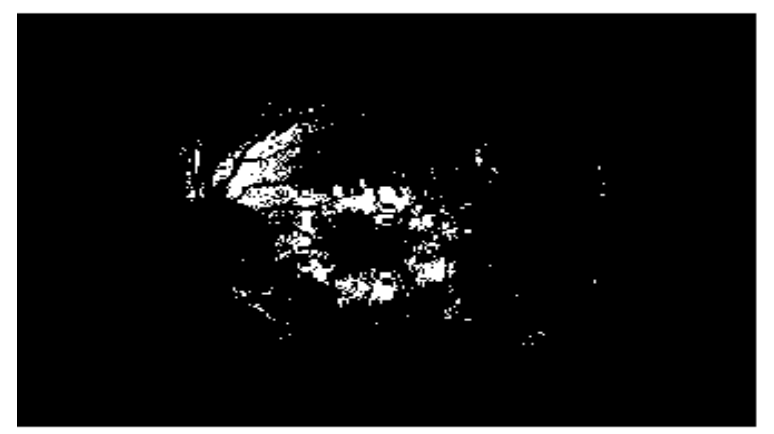

(iii) Quadratic

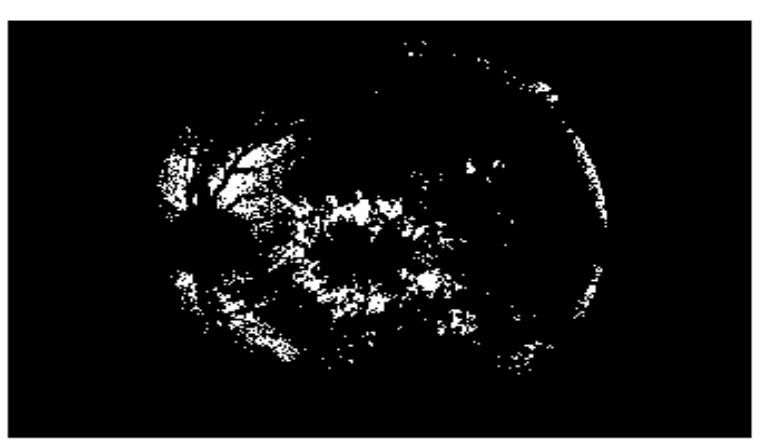

(ii) Polynomial

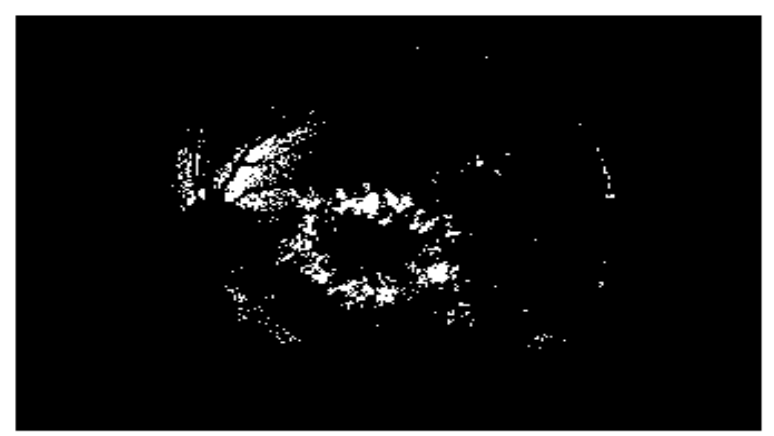

(iv) $\mathrm{RBF}$

Fig.8. Black and white masking image of Fig. 3(i) under varying kernel functions

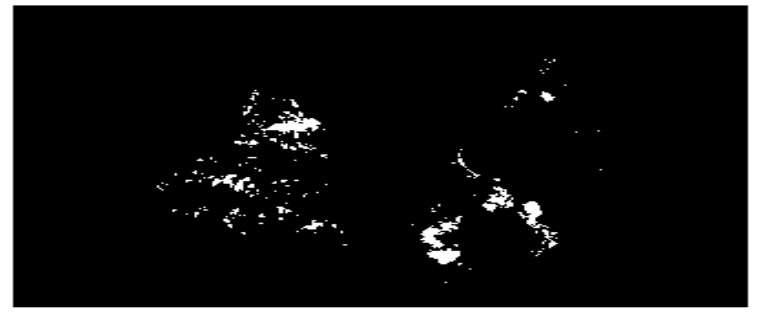

(i) Linear

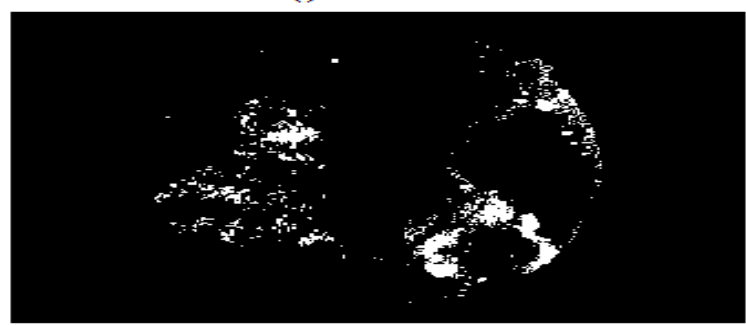

(iii) Quadratic

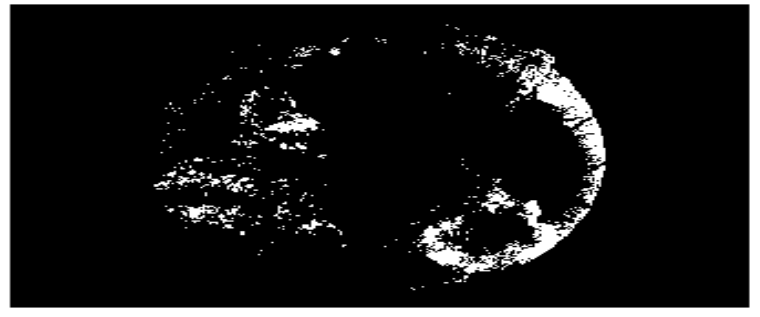

(ii) Polynomial

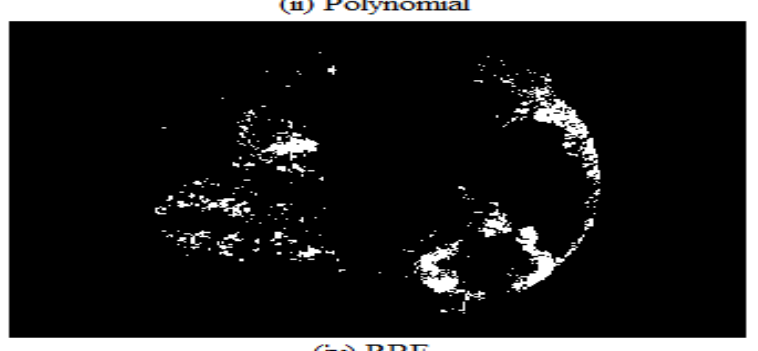

(iv) RBF

Fig.9. Black and white masking image of Fig. 3(ii) under varying kernel functions 


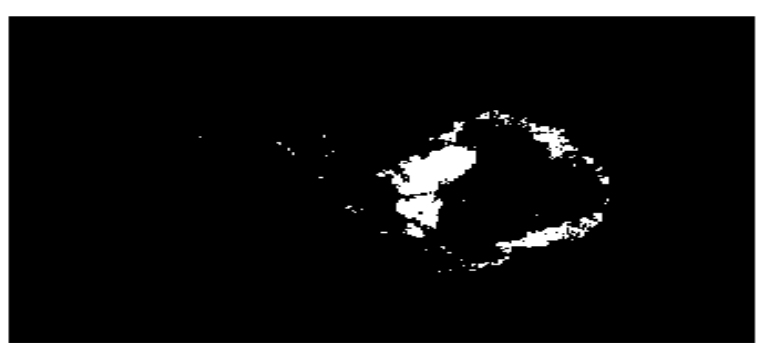

(i) Linear

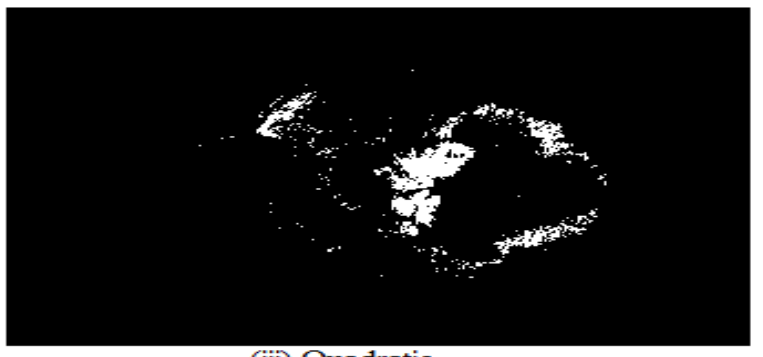

(iii) Quadratic

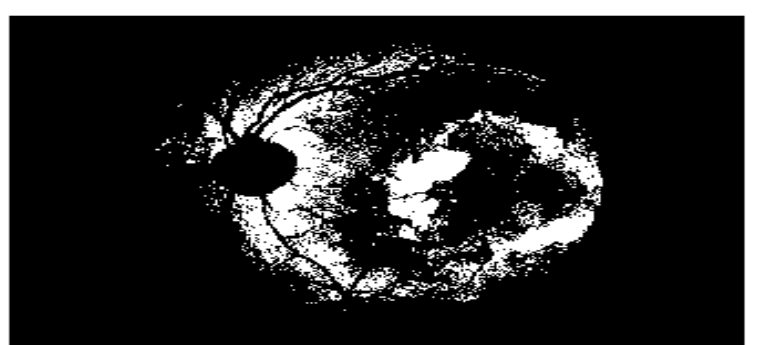

(ii) Polynomial

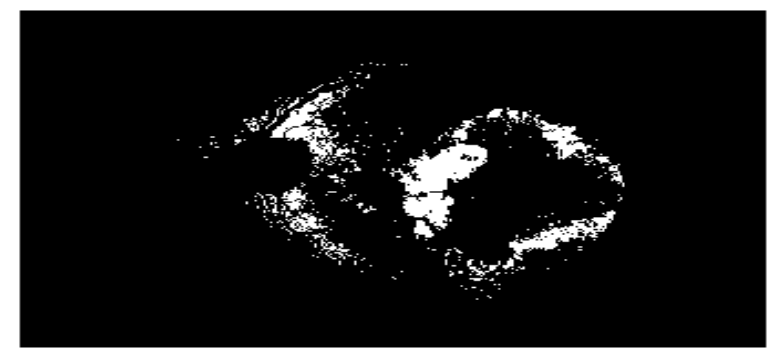

(iv) $\mathrm{RBF}$

Fig.10. Black and white masking image of Fig. 3(iii) under varying kernel functions

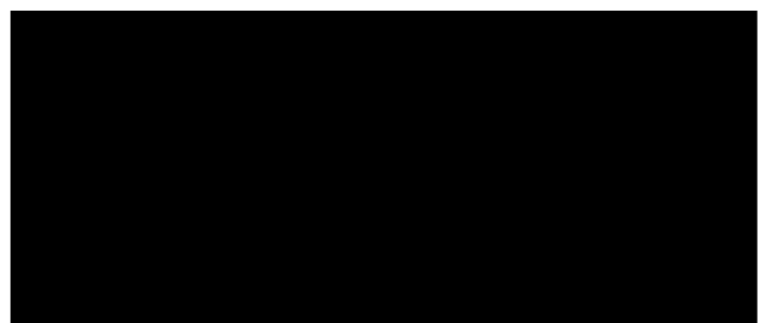

(i) Linear

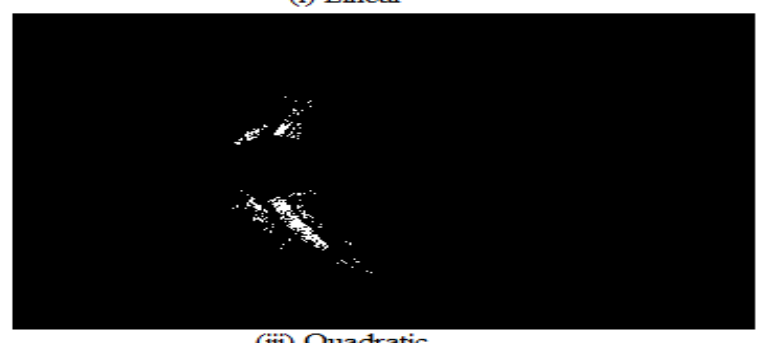

(iii) Quadratic

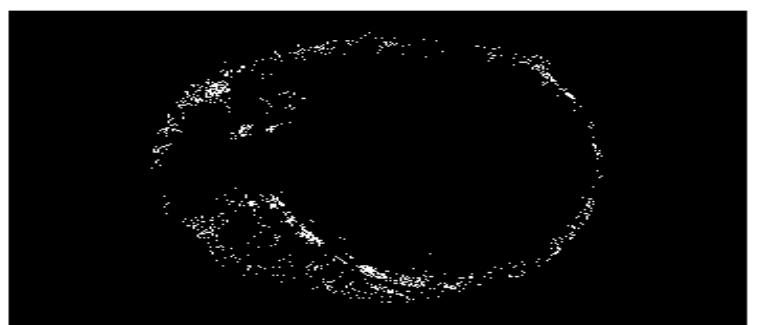

(ii) Polynomial

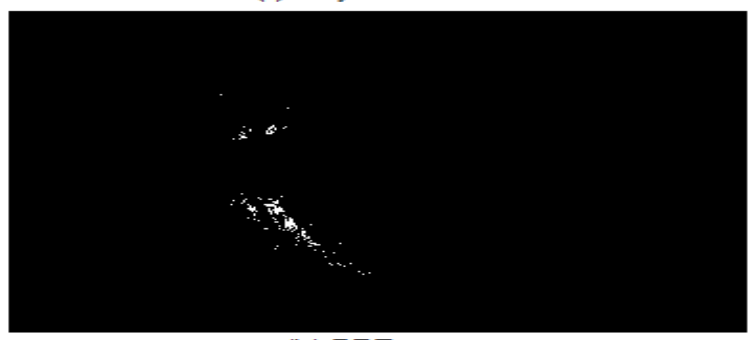

(iv) $\mathrm{RBF}$

Fig.11. Black and white masking image of Fig. 3(iv) under varying kernel functions

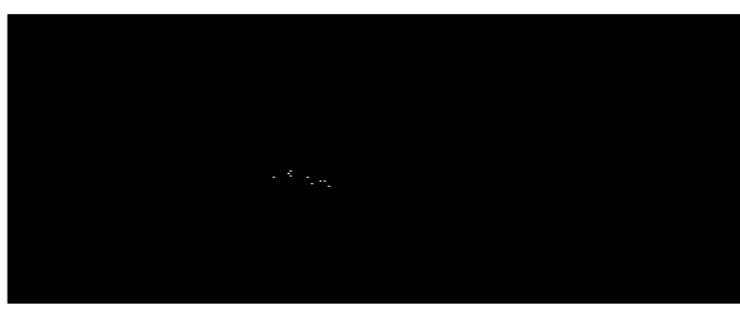

(i) Linear

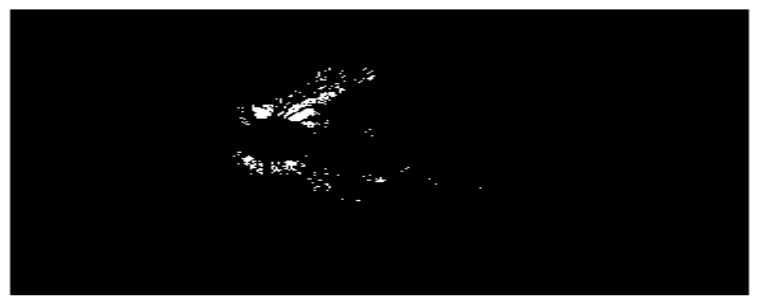

(iii) Quadratic

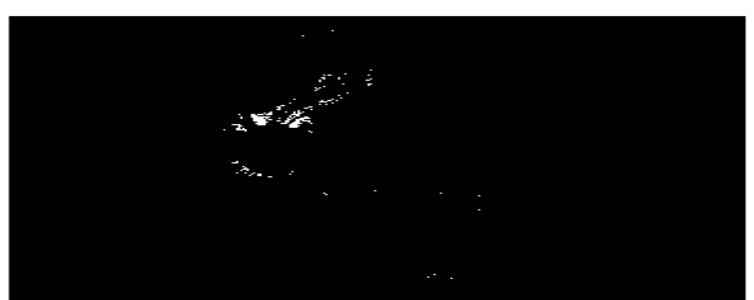

(ii) Polynomial

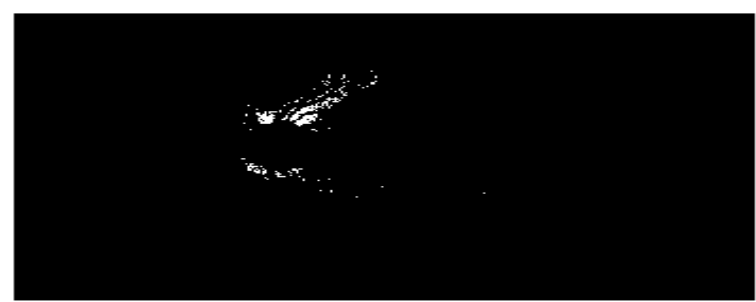

(iv) RBF

Fig.12. Black and white masking image of Fig. 3(v) under varying kernel functions 


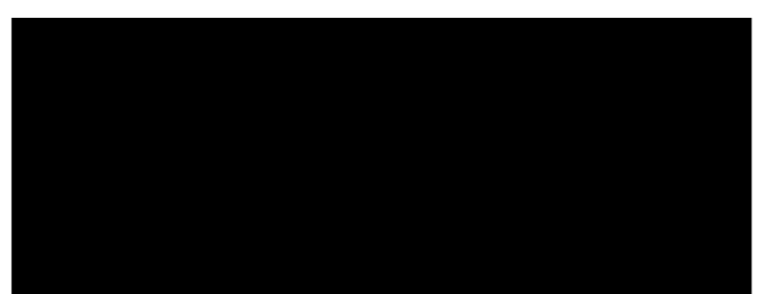

(i) Linear

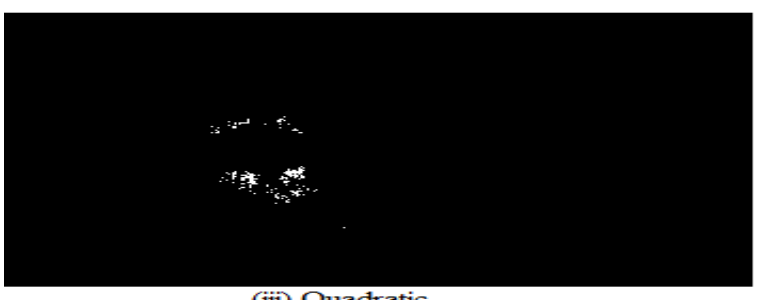

(iii) Quadratic

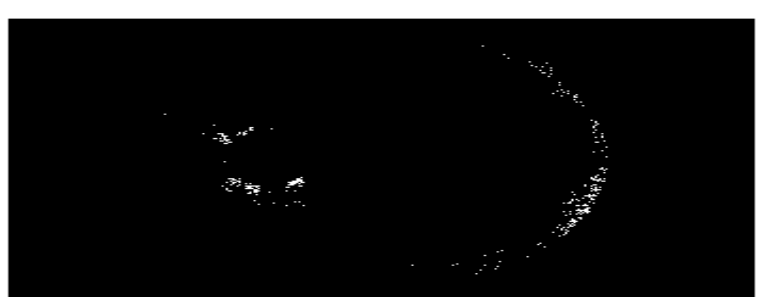

(ii) Polynomial

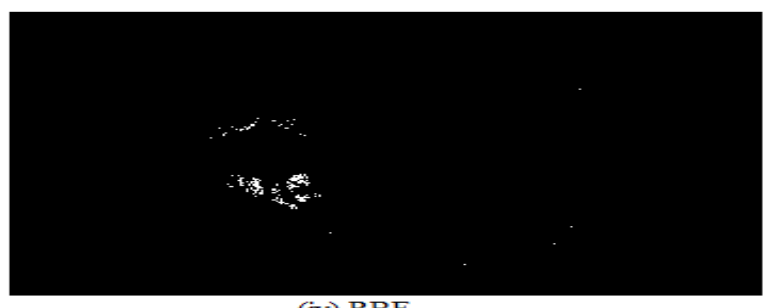

(iv) RBF

Fig.13. Black and white masking image of Fig. 3(vi) under varying kernel functions

Fig. 8 shows the results of black and white masking for exudates fundus image 29. Here, fine exudates segmentation is shown when using linear kernel function compared to others as can be seen in Fig. 8(ii). It clearly followed the Region of Interest (ROI) of exudates areas in image 29. Meanwhile, polynomial, quadratic and RBF kernel function fail to segment the correct exudates areas as shown in Fig. 3(i).

Then, Fig. 9 shows the results of exudates fundus image 131. There are nine ROI of exudates areas that need to be segmented by kernel functions. As can be seen in Fig. 9 (ii), the ROI of exudates areas are very well segmented when applying linear kernel function. However, in polynomial, quadratic and RBF kernel functions show nearly fine exudates segmentation but still they fail to segment the correct exudates areas because the background of the fundus image 131 is segmented as well.

Meanwhile, Fig. 10 shows the black and white masking results for exudates fundus image 175. Here, only one big ROI of exudates area that need to be segmented under varying kernel functions; linear, polynomial, quadratic and RBF. As expected, the linear kernel function shows good exudates segmentation result. Meanwhile polynomial, quadratic and RBF kernel functions still fail to segment the correct exudates areas of fundus image 175 respectively.

Fig. 11 shows the results of black and white masking for normal $I_{w o d}$ image 11. As can be seen, there is no signs of exudates appear in Fig. 11 (ii) when applied linear kernel function. Meanwhile, Fig. 11 (iii), Fig. 11 (iv) and Fig. 11 (v) clearly show false exudates segmentation where they actually segmented the non exudates area and background of the normal $I_{\text {wod }}$ 
image 11 .

Then, Fig. 12 shows the results of normal $I_{\text {wod }}$ image 15. There is supposed no ROI of exudates area that need to be segmented in Fig. 12 (i). But then, the result in Fig. 12 (ii) shows false exudates segmentation where it segmented the areas around OD that appears bright yellow colour. Similarly, Fig. 12 (iii) and Fig. 12 (iv). Then, Fig. 13 (ii), (iii) and (iv) give the results of false exudates segmentation is appeared around OD areas. They are obviously segmented the non exudates area together with additional background of Fig. 3 (vi). As can be seen, the kernel function settings have an effect on the classification results. For SVM1, it was observed that the best parameter in classifying pixels is linear kernel function because it can extract exudates and non exudates candidates efficiently.

The linear kernel function from SVM1 classifier is applied to test all $149 \mathrm{I}_{\text {wod }}$ fundus images; 68 exudates and 81 normal fundus images.

\subsection{Visualization Using CAC and Radar Chart}

Fig. 14 shows the CAC plots for exudates fundus image 132. The CAC plot for Fig. 14 (i) is shown in Fig. 14 (ii). Meanwhile, Fig. 14 (iv) shows CAC plot for invert mask $\mathrm{I}_{\mathrm{wl}}$ fundus image in Fig. 14 (iii). The x-axis represents 64 bit colour map of CAC and y-axis is the correlation vectors. The solid blue line represents the CAC features of Fig. 14 (i) respectively. The dotted red line represents the CAC features of invert mask $\mathrm{I}_{\mathrm{wl}}$ fundus image for Fig. 14 (iii). Once the colour of each pixel (Ci) in Fig. 14 (i) and Fig. 14 (iii) is similar with the 64 bit colour map $(\mathrm{Cm})$, Ci finds colour similarity with pixel neighbours $(\mathrm{Cj})$ around it. If the colour of $\mathrm{Cj}$ same with $\mathrm{Ci}$, the correlation vector is produced. Both situations can be seen in CAC plots Fig. 14 (ii) and Fig. 14 (iv). Since it is hard to distinguish the pattern of CAC plots in Fig. 14 (ii) and Fig. 14 (iv), they were plotted into the same radar chart to visualize the distinguishable features as shown in Fig. 16 (ii). 


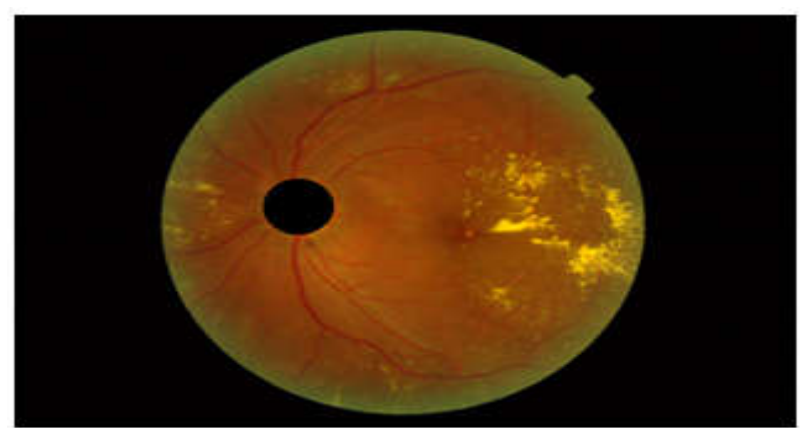

(i) $\quad I_{\text {rod }}$ fundus image 132

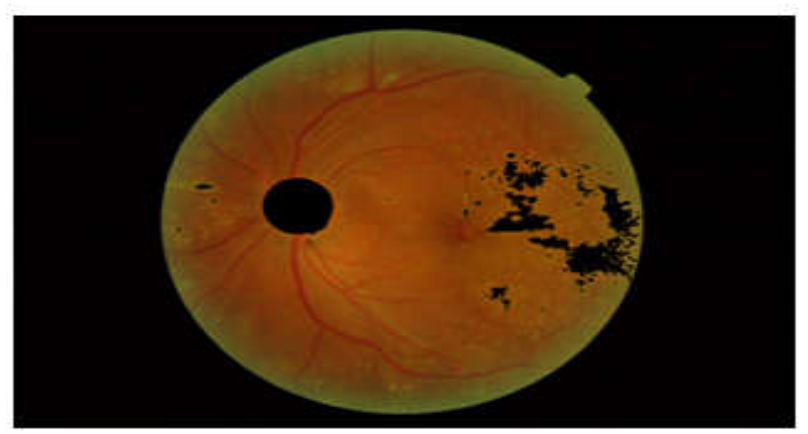

(iii) Invert mask $\mathbf{I}_{w 1}$ fundus image 132

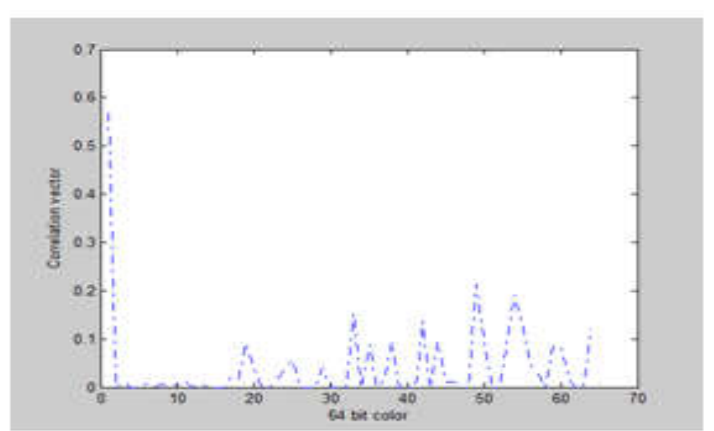

(ii) CAC plot for $I_{\text {wod }}$ fundus image 132

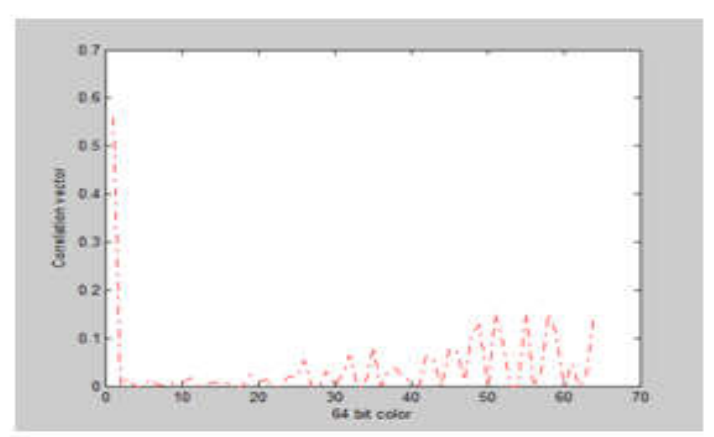

(iv) CAC plot for invert mask $I_{\text {wod }}$ fundus image 132

Fig.14. Sample of CAC plots for exudates fundus image 132

Meanwhile, Fig. 15 shows the CAC plots for normal fundus image 170. Fig. 15 (ii) shows the CAC plot for Fig. 15 (i). Then, the CAC plot for Fig. 15 (iii) is shown in Fig. 15 (iv). The solid blue line represents the CAC features of Fig. 15 (i) respectively. The dotted red line represents the CAC features of invert mask $I_{w l}$ fundus image for Fig. 15 (iii). The $x$-axis represents 64 bit colour map of CAC and y-axis is the correlation vectors. Once the colour of each pixel (Ci) in Fig. 15 (i) and Fig. 15 (iii) same with the 64 bit colour map (Cm), Ci will find colour similarity with pixel neighbours $(\mathrm{Cj})$ around it. If the colour of $\mathrm{Cj}$ same with $\mathrm{Ci}$, the correlation vector is arise. The other way around, the correlation vector is falling almost to zero value. The both situations can be seen in CAC plots Fig. 15(ii) and Fig. 15(iv). For normal fundus image, it is difficult to see the pattern similarity of both CAC plots in Fig. 15(ii) and Fig. 15(iv). So, they were plotted into the same radar chart to visualize the features similarity as shown in Fig. 16(i). 


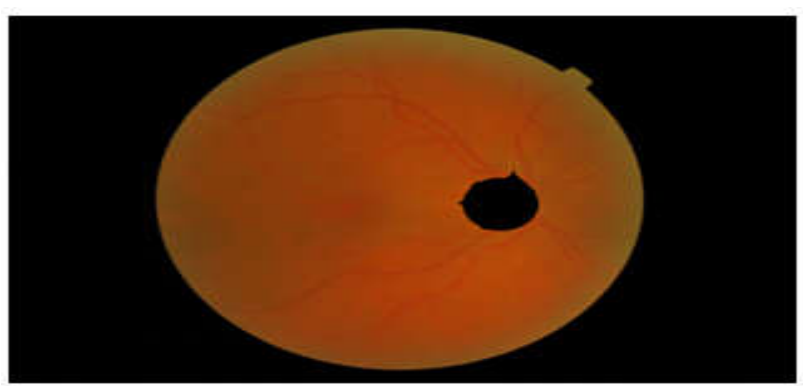

(i) I $_{\text {wod }}$ fundus image 170

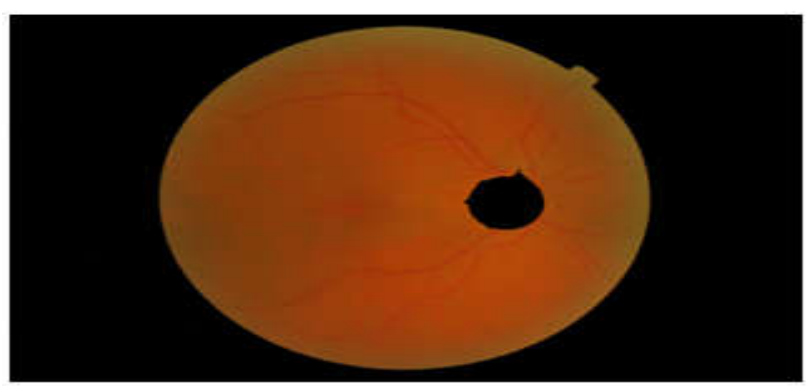

(iii) Invert mask $I_{\mathrm{w} 1}$ fundus image 170

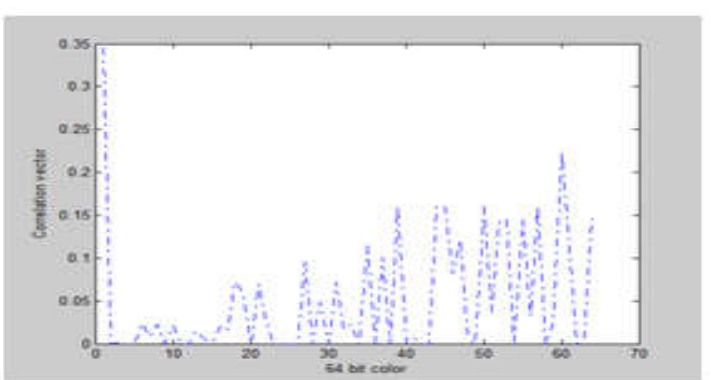

(ii) CAC plot for $I_{\text {wad }}$ fundus image 170

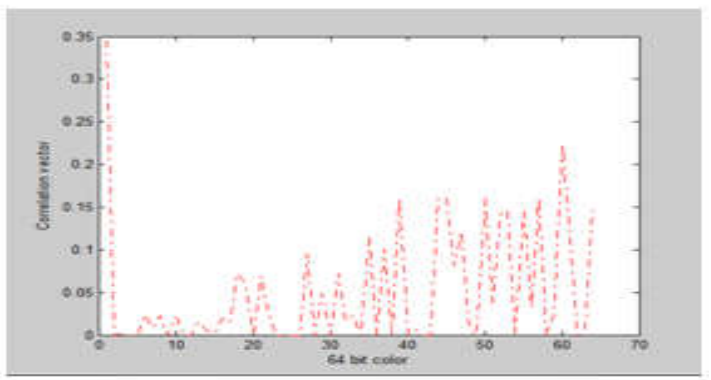

(iv) CAC plot for invert mask $I_{\text {wod }}$ fundus image 170

Fig.15. Sample of CAC plots for normal fundus image 170

Once the CAC features were obtained from $\mathrm{I}_{\mathrm{wod}}$ and invert mask $\mathrm{I}_{\mathrm{wl}}$ fundus images as shown in Fig. 14 and Fig. 15, they were plotted into the same radar chart to visualize the colour features. Fig. 16 shows visualization radar chart for Fig. 14 and Fig. 15. For normal fundus image 170 in Fig. 15, the visualization of radar chart is shown in Fig. 16 (i). As can be seen, the CAC plots are overlap each other meaning no exudates are present. Fig. 16 (ii) shows the visualization of radar chart for exudates fundus image 132 in Fig. 14. Comparatively, when exudates are present, both lines appear to distinguish each other respectively. This is because when exudates are present, the CAC features change as exudates are removed, while for the normal images, the resulting CAC features are similar as no exudates areas are being removed.

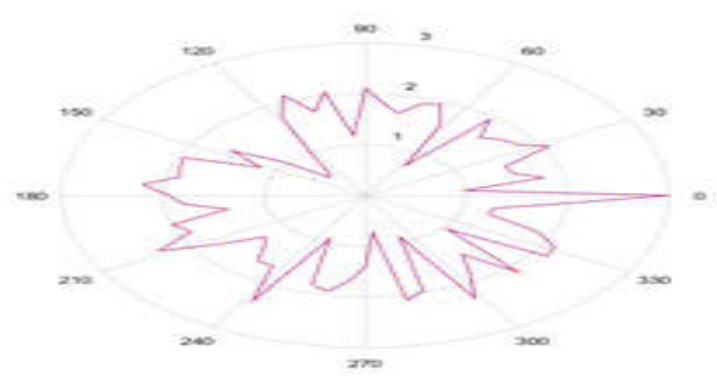

(i)

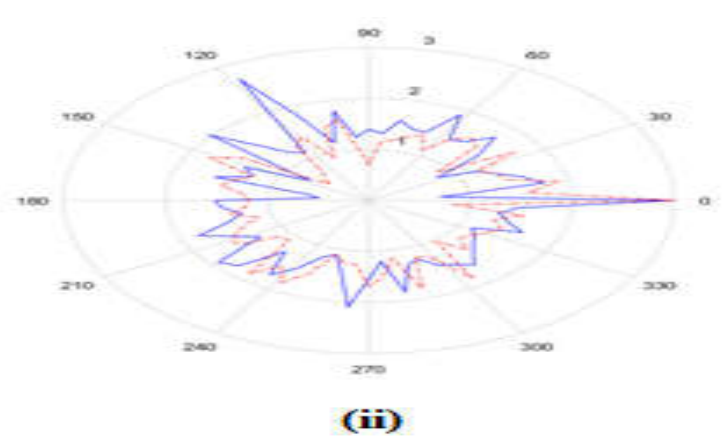

(ii)

Fig.16. Visualization of radar chart for; (i) Radar chart for normal fundus image 170 and (ii) Radar chart for exudates fundus image 132 
Therefore, Table 4 shows number of success visualization of a non-overlapping features percentage for exudates present is $80.8 \%(55 / 68)$ and percentage overlapping features for normal image is $82.7 \%(67 / 81)$.

Table 4. Number of success visualization using CAC and radar chart by using SVM1 classifier

\begin{tabular}{ccc} 
& \multicolumn{3}{c}{ 149 Fundus Images } \\
& Exudates (68) & Normal (81) \\
\hline Overlapping & $13 / 68$ & $67 / 81$ \\
Non Overlapping & $55 / 68$ & $14 / 81$ \\
\hline
\end{tabular}

\subsection{Classification Using SVM2}

The SVM2 classifier was applied to 149 visualization results to determine the best optimal parameters for the highest accuracy of its. A summary of classification results for SVM2 is shown in Table 5 and Table 6.

According to Table 5, it shows classification accuracy results of varied $\mathrm{C}$ values. The value is varied from $0.01,0.1,1$ and 10 to get the best classification accuracy to be used in SVM2. From Table 5, the suitable value for $\mathrm{C}$ is equal to 1 since it accurateness is $98.5 \%$ which is the highest than others.

There are four types of kernel functions applied for pixels classification test; linear, polynomial, quadratic and RBF. As can be seen in Table 6, RBF kernel functions show excellent classification [48] accuracy which is above $96 \%$ compare to other kernel functions.

Table 5. Regularization parameter (C) and classification accuracy for SVM2

\begin{tabular}{cc}
\hline Regularization Parameter $(\mathbf{C})$ & Classification Accuracy \\
\hline $\mathbf{0 . 0 1}$ & $\mathbf{9 6 . 1 \%}$ \\
0.1 & $97.4 \%$ \\
1 & $98.5 \%$ \\
10 & $95.9 \%$ \\
\hline
\end{tabular}

Table 6. SVM2 kernel function and CAC features classification 


\begin{tabular}{cc}
\hline Kernel Function & Classification Accuracy (\%) \\
\hline Linear & Training $=96 \%$ \\
Testing $=72.97 \%$ \\
Polynomial & Training $=94.67 \%$ \\
Testing $=72.97 \%$ \\
Quadratic & Training $=89.33 \%$ \\
RBF & Testing $=66.22 \%$ \\
& Training $=96 \%$ \\
& Testing $=82.43 \%$ \\
\hline
\end{tabular}

\section{CONCLUSION}

In conclusion, by using linear kernel function for SVM1, a percentage for exudates fundus images is $80.8 \%(55 / 68)$ and percentage for normal fundus image is $82.7 \%(67 / 81)$. From this research, the variations of fundus images caused by retinal pigmentation, acquisition angle and illumination are the factors for wrong exudates detection in fundus images. The brightness also known as illumination in fundus images appears to be of such poor quality as to interfere with analysis. Also, the retinal pigmentation and acquisition angles of OD.

Then, the accuracy for visualization results is $96 \%$ by using RBF kernel function for SVM2 classifier which is nonlinear model that suitable for complex case. Therefore, the visualization characteristics of normal and exudates fundus images using the proposed methods have been proven to diagnose DR.

\section{ACKNOWLEDGEMENTS}

This research is funded by Institute of Research Management and Innovation (IRMI), Universiti Teknologi MARA (UiTM) Selangor, Malaysia under Grant No: 600-RMI/DANA 5/3/PSI (195/2013). The authors wish to thank faculty of Electrical Engineering UiTM Shah Alam Selangor for all the support given during this research and Universiti Selangor (UNISEL) for the scholarship awarded to the first author. 


\section{REFERENCES}

[1] Acharya U R, Lim C M, Ng E Y, Chee C, Tamura T. Computer-based detection of diabetes retinopathy stages using digital fundus images. Proceedings of the Institution of Mechanical Engineers, Part H: Journal of Engineering in Medicine, 2009, 223(5):545-553

[2] Kovács L, Kurtán B, Eigner G, Rudas I, Chui C K. Analysis of a novel time-delay diabetes model. In IEEE European Control Conference, 2015, pp. 13-18

[3] Rahaman S. Diabetes diagnosis decision support system based on symptoms, signs and risk factor using special computational algorithm by rule base. In IEEE 15th International Conference on Computer and Information Technology, 2012, pp. 65-71

[4] Olokoba A B, Obateru O A, Olokoba L B. Type 2 diabetes mellitus: A review of current trends. Oman Medical Journal, 2012, 27(4):269-273

[5] Elahi-Moghaddam Z, Behnam-Rassouli M, Mahdavi-Shahri N, Hajinejad-Boshroue R, Khajouee E. Comparative study on the effects of type 1 and type 2 diabetes on structural changes and hormonal output of the adrenal cortex in male Wistar rats. Journal of Diabetes and Metabolic Disorders, 2013, 12(1):1-6

[6] Pratley R E. The early treatment of type 2 diabetes. American Journal of Medicine. 2013, 126(9):2-9

[7] Kam L Y. Rawat diabetes lebih awal. Kuala Lumpur: Utusan Malaysia, 2011

[8] Nentwich M M, Ulbig M W. Diabetic retinopathy-Ocular complications of diabetes mellitus. World Journal of Diabetes 2015, 6(3):489-499

[9] He Z, King G L. Microvascular complications of diabetes. Endocrinology and Metabolism Clinics of North America, 2004, 33(1):215-238

[10] Bala M P, Vijayachitra S. Computerised retinal image analysis to detect and quantify exudates associated with diabetic retinopathy. International Journal of Computer Applications, 2012, 54(2):7-12

[11] Ooyub S, Ismail F, Daud N. Diabetes program in Malaysia-Current and future. NCD Malaysia, 2004, 3(2):6-12 
[12] Gadriye D, Khandale G. Neural Network based method for the diagnosis of diabetic retinopathy. In IEEE International Conference on Computational Intelligence and Communication Networks, 2014, pp. 1073-1077

[13] Dutta M K, Ganguly S, Srivastava K, Ganguly S, Parthasarathi M, Burget R, Masek J. An efficient grading algorithm for non-proliferative diabetic retinopathy using region based detection. In 38th IEEE International Conference on Telecommunications and Signal Processing, 2015, pp. 743-747

[14] Ganguly S, Ganguly S, Srivastava K, Dutta M K, Parthasarathi M, Burget R, Riha K. An adaptive threshold based algorithm for detection of red lesions of diabetic retinopathy in a fundus image. In IEEE International Conference on Medical Imaging, m-Health and Emerging Communication Systems, 2014, pp. 91-94

[15] Silvia R C, Vijayalakshmi R. Detection of non-proliferative diabetic retinopathy in fundus images of the human retina. In IEEE International Conference on Information Communication and Embedded Systems, 2013, pp. 978-983

[16] Das H, Saha A, Deb S. An expert system to distinguish a defective eye from a normal eye. In IEEE International Conference on Issues and Challenges in Intelligent Computing Techniques, 2014, pp. 155-158

[17] Tjandrasa H, Putra R E, Wijaya AY, Arieshanti I. Classification of non-proliferative diabetic retinopathy based on hard exudates using soft margin SVM. In IEEE International Conference on Control System, Computing and Engineering, 2013, pp. 376-380

[18] Subbuthai P, Muruganand S. Restoration of retina images using extended median filter algorithm. In 2nd IEEE International Conference on Signal Processing and Integrated Networks, 2015, pp. 131-138

[19] Boyd K. Retinal detachment: What is a torn or detached retina? California: American Academy of Ophthalmology, 2016

[20] MaheshK K, Nilesh S K. Review on fundus image acquisition techniques with data base reference to retinal abnormalities in diabetic retinopathy. International Journal of Computer Applications, 2013, 68(8):17-27 
[21] Udayakumar K, Bharati P P, Verma S, Nath M K. Automatic estimation of vision degradation from color fundus image. International Journal of Image, Graphics and Signal Processing, 2015, 7(11):26-34

[22] Klein J C, Menard M, Cazuguel G, Fernandez-Maloigne C, Shaefer G, Gain P, Cochener B, Massin P, Lay B, Charton B. Methods to evaluate segmentation and indexing techniques in the field of retinal ophthalmology (MESSIDOR). Paris: MINES ParisTech, 2016

[23] Yu H, Barriga E S, Agurto C, Echegaray S, Pattichis M S, Bauman W, Soliz P. Fast localization and segmentation of optic disk in retinal images using directional matched filtering and level sets. IEEE Transactions on Information Technology in Biomedicine, 2012, 16(4):644-657

[24] Hassan H A, Tahir N M, Yassin I, Zabidi A, Yahaya C H, Shafie S M. Automated optic disc removal in fundus images using iterative heuristics and morphological operations. In IEEE Conference on Systems, Process and Control, 2013, pp. 230-233

[25] Lim S T, Zaki W M, Hussain A, Lim S L, Kusalavan S. Automatic classification of diabetic macular edema in digital fundus images. In IEEE Colloquium on Humanities, Science and Engineering, 2011, pp. 265-269

[26] Aquino A, Gegúndez-Arias M E, Marín D. Detecting the optic disc boundary in digital fundus images using morphological, edge detection, and feature extraction techniques. IEEE Transactions on Medical Imaging, 2010, 29(11):1860-1869

[27] GeethaRamani R, Dhanapackiam C. Automatic localization and segmentation of optic disc in retinal fundus images through image processing techniques. In IEEE International Conference on Recent Trends in Information Technology, 2014, pp. 1-5

[28] Aqeel A F, Ganesan S. Automated algorithm for retinal image exudates and drusens detection, segmentation, and measurement. In IEEE International Conference on Electro/Information Technology, 2014, pp. 206-215

[29] Eadgahi M G, Pourreza H. Localization of hard exudates in retinal fundus image by mathematical morphology operations. In 2nd IEEE International eConference on Computer and Knowledge Engineering, 2012, pp. 185-189 
[30] Rajput G G, Patil P N. Detection and classification of exudates using k-means clustering in color retinal images. In 5th IEEE International Conference on Signal and Image Processing, 2014, pp. 126-130

[31] Jaafar H F, Nandi A K, Al-Nuaimy W. Automated detection of exudates in retinal images using a split-and-merge algorithm. In 18th IEEE European Signal Processing Conference, 2010, pp. 1622-1626

[32] Narang A, Narang G, Singh S. Detection of hard exudates in colored retinal fundus images using the Support Vector Machine classifier. In 6th IEEE International Congress on Image and Signal Processing, 2013, pp. 964-968

[33] Ashraf M N, Habib Z, Hussain M. Texture feature analysis of digital fundus images for early detection of diabetic retinopathy. In 11th IEEE International Conference on Computer Graphics, Imaging and Visualization, 2014, pp. 57-62

[34] Roberts J C, Ritsos P D, Badam S K, Brodbeck D, Kennedy J, Elmqvist N. Visualization beyond the desktop-The next big thing. IEEE Computer Graphics and Applications, 2014, $34(6): 26-34$

[35] Yan Z, Liqun Y, Lei W. A research for the classification of knowledge visualization. In IEEE International Conference on Electrical and Control Engineering, 2011, pp. 6235-6238

[36] Chen M, Ebert D, Hagen H, Laramee R S, Van Liere R, Ma K L, Ribarsky W, Scheuermann G, Silver D. Data, information, and knowledge in visualization. IEEE Computer Graphics and Applications, 2009, 29(1):12-19

[37] Goebel R. A sketch of a theory of visualization. In IEEE International Conference on Information Visualization Theory and Applications, 2014, pp. 218-221

[38] Van Wijk J J. Views on visualization. IEEE Transactions on Visualization and Computer Graphics, 2006, 12(4):421-432

[39] Ruchikachorn P, Mueller K. Learning visualizations by analogy: Promoting visual literacy through visualization morphing. IEEE Transactions on Visualization and Computer Graphics, 2015, 21(9):1028-1044 
[40] Borkin M A, Vo A A, Bylinskii Z, Isola P, Sunkavalli S, Oliva A, Pfister H. What makes a visualization memorable? IEEE Transactions on Visualization and Computer Graphics, 2013, 19(12):2306-2315

[41] Singh S R, Kohli S. Enhanced CBIR using color moments, HSV histogram, color auto correlogram, and gabor texture. International Journal of Computer Systems, 2015, 2(5):161-165

[42] Shen X, Wang X, Du J. A novel image retrieval method based on color autocorrelogram and mutual information. In International Conference on Advanced Computer Science and Electronics Information, 2013, pp. 587-590

[43] Talib A, Mahmuddin M, Husni H, George L E. Efficient, compact, and dominant color correlogram descriptors for content-based image retrieval. In 5th International Conferences on Advances in Multimedia, 2013, pp. 1-10

[44] Mustaffa M R, Ahmad F, Mahmod R, Doraisamy S. Multi-resolution joint auto correlograms: Determining the distance function. In IEEE International Conference on Information Retrieval and Knowledge Management, 2012, pp. 198-202

[45] Huang J, Zabih R. Combining color and spatial information for content-based image retrieval. In European Conference on Digital Libraries, 1998, pp. 1-5

[46] Gudivada V N, Raghavan V V. Content based image retrieval systems. Computer, 1995, 28(9):18-22

[47] Sahak R, Mansor W, Lee K Y, Zabidi A, Yassin A I. Optimization of principal component analysis and support vector machine for the recognition of infant cry with asphyxia. International Journal of Computers and Applications, 2013, 35(3):99-107

[48] Hasan C Z, Jailani R, Tahir N M, Yassin I M, Rizman Z I. Automated classification of autism spectrum disorders gait patterns using discriminant analysis based on kinematic and kinetic gait features. Journal of Applied Environmental and Biological Sciences, 2017, $7(1): 150-156$

\section{How to cite this article:}

Hassan HA, Tahir NM, Zabidi A, Yassin IM, Karbasi M. Classification of visualization exudates fundus images results using support vector machine. J. Fundam. Appl. Sci., 2017, 9(4S), $19-44$. 